\title{
Metastatic choriocarcinoma presenting as multiple intracerebral haemorrhages: the role of imaging in the elucidation of the pathology
}

\author{
D Kidd, G T Plant, F Scaravilli, A C E McCartney, M Stanford, E M Graham
}

\section{Case report}

A 48 year old local government officer was admitted to the Medical Eye Unit of St Thomas' Hospital after the sudden onset 13 days previously of blurred vision with headache. Investigation at another hospital had disclosed a right homonymous central scotoma, and brain CT had shown haemorrhages in both occipital lobes.

There had been no previous neurological symptoms and no other symptoms were noted on systematic enquiry. On examination he was afebrile and there was no lymphadenopathy or organ enlargement. Examination of the heart, lungs, joints, and abdomen was normal. There were no skin lesions. Neurological examination was normal apart from the right homonymous central scotoma. The fundi were normal.

Erythrocyte sedimentation rate was 51, haemoglobin $13.5 \mathrm{~g} / \mathrm{dl}$, white cell count 13.4 , and biochemical screening was normal. Brain MRI showed eight haemorrhagic lesions of varying age throughout both hemispheres (fig 1). Chest radiography showed three coin lesions. An MR angiogram of the large intracranial vessels was normal and a four vessel arteriogram did not show any vascular abnormality or tumour circulation. An echocardiogram, coagulation studies, antinuclear, extract-

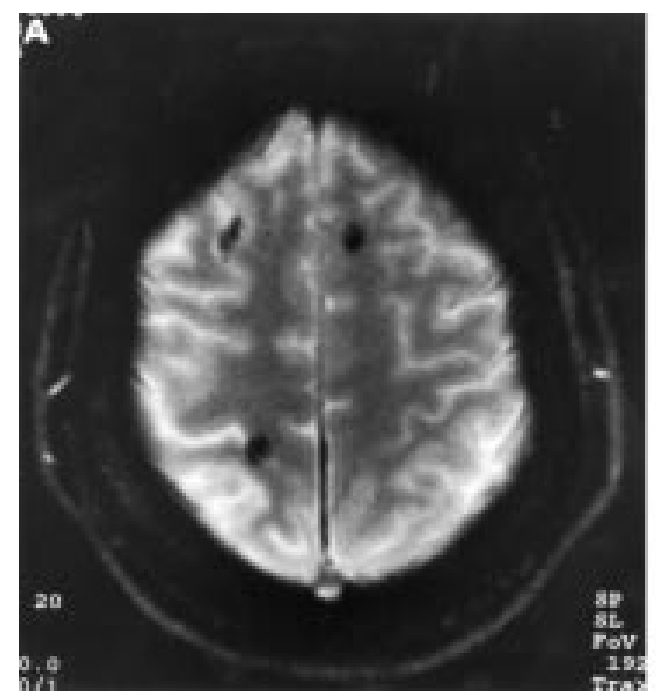

able nuclear antibodies, and ANCA were negative, and complement studies were normal.

Seven days after admission he developed pleuritic chest pain and a cough productive of blood stained sputum. A ventilation-perfusion scan of the lungs showed multiple matched defects throughout both lung fields. Haemoglobin fell to 10.7 and erythrocyte sedimentation rate rose to 102 . Cytology of sputum disclosed abnormal but not malignant cells. Thorax CT disclosed multiple ill defined nodules of varying size throughout both lung fields and no hilar or mediastinal lymphadenopathy. Fine needle aspiration of one of these lesions showed non-small cell carcinoma cells.

Over the course of the next 10 days he deteriorated, with increasing breathlessness, and the appearances of the chest radiograph became more pronounced. He received supportive therapy with oxygen and blood transfusion. An ${ }^{18}$ F-fluorodeoxyglucose (FDG) PET scan of the body showed increased isotope uptake in both lungs, the left shoulder, and an area below the bladder, thought to be either testis or bowel). An ultrasound of the testes was normal; sigmoidoscopy showed no mucosal lesion. Brain ${ }^{18}$ FDG PET showed decreased uptake of isotope in both occipital lobes corre-

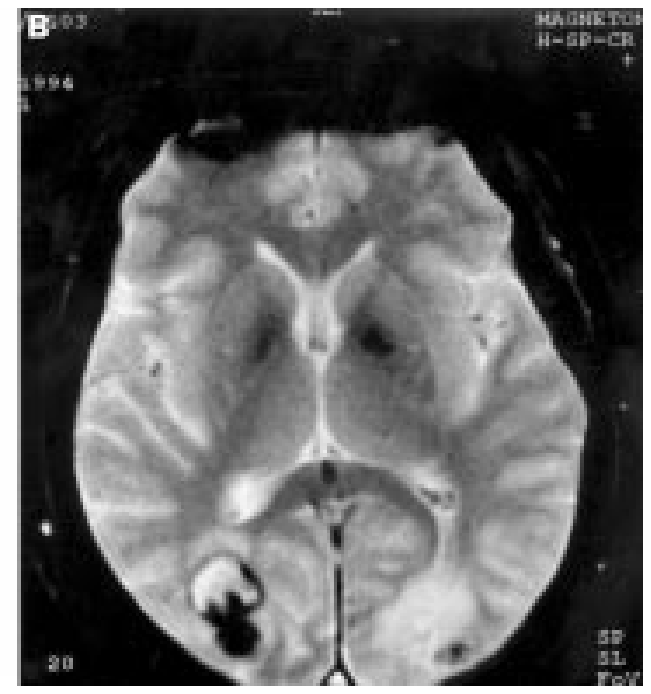

Figure 1 ( $A, B)$ T2 weighted (TR 5000 ms TE 90 ms) MRI showing multiple haemorrhages of varying age throughout both hemispheres.

\author{
Department of
Histopathology, St
Thomas' Hospital,
Lambeth Palace Road,
London, UK
A C E McCartney,
deceased
Department of
Neuropathology,
Institute of Neurology,
Queen Square,
London, UK
F Scaravilli
Correspondence to:
Dr EM Graham, Consultant
Ophthalmologist, The
Medical Eye Unit, St
Thomas' Hospital, Lambeth
Palace Road, London SE1
7EH, UK. Telephone 0044
171 928 9292.
Received 23 February 1998
and in revised form
22 May 1998
Accepted 1 June 1998 \\ D Kidd \\ G T Plant \\ M Stanford \\ E M Graham
}



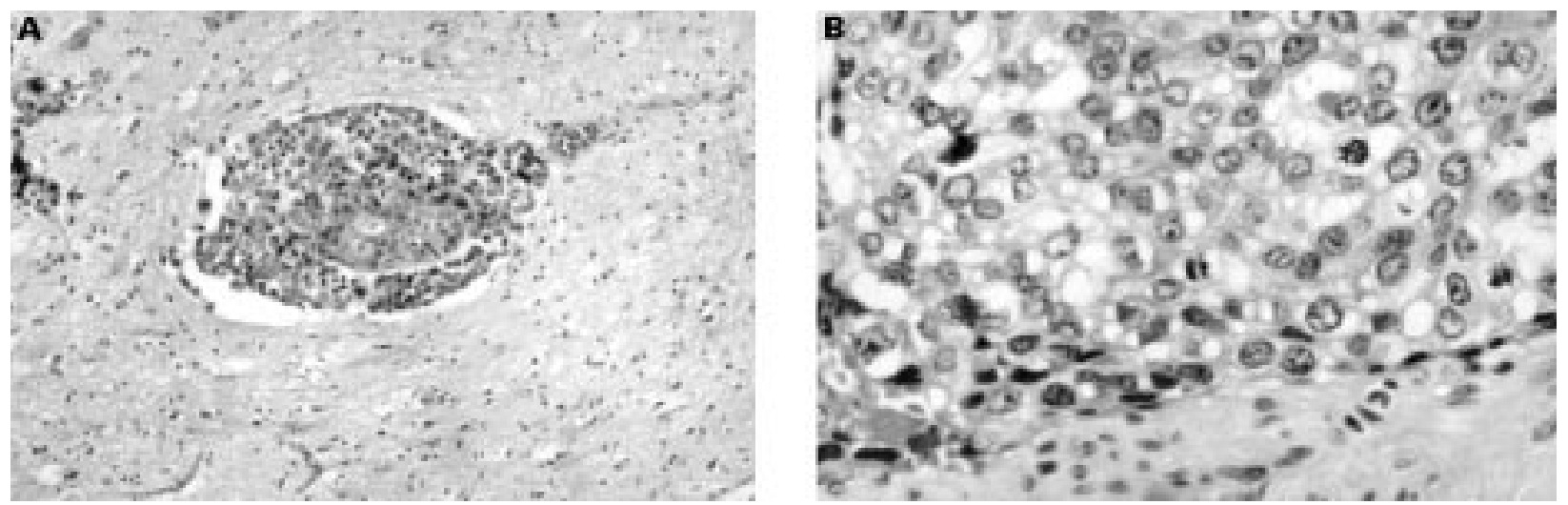

Figure 2 (A) Photomicrograph of brain showing perivascular space distended by a proliferation of neoplastic cells. Other, smaller clumps are seen elsewhere (arrow). The surrounding tissue shows mild gliosis. Haematoxylin and eosin, originally $\times 120$. (B) Photomicrograph showing the appearances of the tumour cells. Note the presence of two mitotic figures. Haematoxylin and eosin, originally $\times 360$.

sponding to the sites of the two haemorrhagic lesions seen on MRI, but no uptake by the lesions themselves as would typically be seen in cerebral metastasis. ${ }^{1}$

An open lung biopsy was performed. After this he required postoperative ventilation. The diagnosis was made histologically and treatment commenced, but he developed recurrent episodes of asystole and died.

\section{Pathology}

Only the brain was examined at postmortem; it weighed $1500 \mathrm{~g}$. The skull was normal and the dura mater was attached by several adhesions to the brain. The left occipital lobe showed a soft, haemorrhagic lesion, and several other lesions were seen when the brain was cut.

Histological examination of tissue sections taken from the brain showed numerous clumps of anaplastic malignant cells in cerebral vessels with evidence of surrounding haemorrhage and intramural thrombus, and tumour cells outside the endothelial surface within the Virkow-Robin space (fig 2A). The appearances of the tumour within the brain were similar to those in the lung and showed anaplastic tumour suggestive of metastatic trophoblastic teratoma (fig 2B), with numerous foci of tumour cells within the interstitium and in blood vessels. Immunocytochemical stains were strongly positive for pankeratin and placental alkaline phosphatase and $\beta$-HCG.

\section{Discussion}

Choriocarcinoma is a rare germ cell tumour in males, accounting for only $4 \%$ of testicular tumours. ${ }^{2}$ The tumour is characterised by the presence of two cell lines: the cytotrophoblast, the cells of which lie in sheets to form villus-like structures, and the syncytiotrophoblast, which secretes $\beta$-human chorionic gonadotrophin and human placental lactogen and is seen at the advancing edge of the tumour. In females, choriocarcinoma is more readily diagnosed, although it remains a very rare form of malignancy; $50 \%$ occur after hydatidiform mole, $25 \%$ after spontaneous abortion, and $22.5 \%$ after normal pregnancies. Rarely it may follow ectopic pregnancy and may arise from the ovaries. In persons of either sex, it may arise in the mediastinum and in the region of the pineal gland. The tumour has a marked tendency to metastasise early, by blood-borne dissemination, in particular to the lungs. The disease often presents with symptoms related to metastatic spread, as the primary tumour may remain very small.

Cerebral haemorrhage has been reported to complicate metastatic choriocarcinoma; brain metastases are thought to arise from tumour emboli from the lungs, as it is most uncommon for such lesions to arise in the absence of pulmonary metastases. ${ }^{3}$ Patients tend to present with a clinical picture compatible with cerebral haemorrhage, with headache, seizure, focal neurological deficits, and obtundation. Haemorrhage is common because of the innate capacity of trophoblastic cells to invade and erode vessel walls. No tumour circulation is established and this explains why in our case no abnormality was detected on cerebral angiography and also why there was a reduced rather than the expected increase in isotope uptake on PET. Both the absence of a tumour circulation on angiography and a lack of ${ }^{18} \mathrm{FDG}$ uptake on PET may argue against a diagnosis of cerebral metastasis from other tumours. In choriocarcinoma single or multiple aneurysms are sometimes seen, although rarely, and tend to arise from peripheral branches of the main cerebral arteries. ${ }^{45}$ Such aneurysms are postulated to occur because of partial destruction of the vessel wall occurring after necrotic tumour embolus lodges in the vessel.

Intracerebral haemorrhage is particularly common with choriocarcinoma, melanoma, bronchial carcinoma, and hypernephroma. ${ }^{7-9}$ Brain haemorrhage associated with metastatic tumour may be the presenting feature of the disease; it tends to have multiple sites and may be associated with surrounding oedema, but in other respects it is difficult to differentiate the imaging appearances from those due to cerebrovascular disease. ${ }^{1011}$

Haemorrhage may also complicate primary brain tumours, particularly ependymoma, glioblastoma multiforme, and oligodendroglioma, ${ }^{8}$ but rarely meningioma. Metastatic tumours may also be associated with subdural haematoma and subarachnoid haemorrhage. Both are associated with cortical lesions which rupture into 
the subdural or subarachnoid space, or into the ventricles. $^{7}$

Other cerebrovascular complications of metastatic carcinoma include infarction due to tumour embolus or to marantic endocarditis, venous sinus thrombosis, or partial occlusion ${ }^{12}$ and complications of coagulation disorders arising from the disease process itself, particularly in reticuloendothelial malignancies. ${ }^{913}$

Gestational choriocarcinoma is highly sensitive to chemotherapy; ${ }^{14}{ }^{15}$ that arising from the testis and ovary is less so but the mortality due to choriocarcinoma in males has fallen from $70 \%$ to $20 \%$ after the introduction of chemotherapy, ${ }^{16}$ principally using actinomycin D, methotrexate, cyclophosphamide, and recently, etoposide. The treatment of choriocarcinoma with cerebral metastases involves chemotherapy in conjunction with cranial irradiation. ${ }^{17-19}$ Surgery is associated with a high incidence of haemorrhagic complications ${ }^{5}$ and should only be performed if the haemorrhage itself is life threatening.

This case emphasises the difficulty which may be encountered in making a diagnosis in haemorrhagic metastasis. Consideration of the pathology allows a more clear understanding of the distinctive clinical and radiological characteristics of metastatic choriocarcinoma.

1 Lewis P, Griffin S, Marsden P, et al. Whole body ${ }^{18} \mathrm{~F}$-fluorodeoxyglucose positron emission tomography in pre-operative evaluation of lung carcinoma. Lancet 1994;344:1265-6.

2 Bredael JJ, Vugrin D, Whitmore WF. Autopsy findings in 154 patients with germ cell tumors of the testis. Cancer 1982;50:548-51.

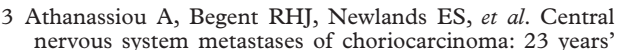
nervous system metastases of choriocarcinoma: 23 years'
experience at Charing Cross Hospital. Cancer 1983;52: experience

4 Pullar M, Blumbergs PC, Phillips GE, et al. Neoplastic cerebral aneurysm from metastatic gestational choriocarcinoma. F Neurosurg 1985;63:644-7.

5 Seigle JM, Caputy AJ, Manz HJ, et al. Multiple oncotic intracranial aneurysms and cardiac metastasis from choriocarcinoma: case report and review of the literature. Neurosurgery 1987;20:39-42.

6 Ho KL. Neoplastic aneurysm and intracranial hemorrhage. Cancer 1982;50:2935-40.

7 Mandybur TI. Intracranial hemorrhage caused by metastatic tumors. Neurology 1977;27:650-5.

8 Wakai S, Yamakawa K, Manaka S, et al. Spontaneous intracranial hemorrhage caused by brain tumor: its incidence and clinical significance. Neurosurgery 1982;10:437-44.

9 Graus F, Rogers LR, Posner JB. Cerebrovascular complications in patients with cancer. Medicine 1985;64:16-35.

10 Sze G, Krol G, Olsen WL, et al. Hemorrhagic neoplasms: MR mimics of occult vascular malformations. AfR Am $\mathcal{F}$ Roentgenol 1987;149:1223-30.

11 Watanabe AS, Smoker WRK. Computed tomography and angiographic findings in metastatic choriocarcinoma. $f$ Comput Assist Tomogr 1989;13:319-22.

12 Plant GT, Donald JJ, Jackowski A, et al. Partial, nonthrombotic, superior sagittal sinus occlusion due to occipital skull tumours. F Neurol Neurosurg Psychiatry 1991;54: $520-3$.

13 Dubas F, Serre I. Complications vasculaires cérébrales des cancers. Rev Neurol 1992;148:663-71.

14 Soper JT. Management of gestational trophoblastic disease. Oncology 1993;7:68-82.

15 Herrington S. Enhancing cure and palliation: radiation therapy in the treatment of metastatic gestational trophoblastic neoplasia. Semin Oncol 1995;22:185-92.

16 Bosl GJ, Gluckman R, Geller NL, et al. VAB-6: an effective chemotherapy regime for patients with germ cell tumours. f Clin Oncol 1986;4:1493-9.

17 Logothetis CJ, Samuels ML, Trindode A. The management of brain metastases in germ cell tumours. Cancer 1982;49: $1278-81$

18 Rustin GJS, Newlands ES, Bagshawe KD, et al. Successful management of metastatic and primary germ cell tumours in the brain. Cancer 1986;57:2108-13.

19 Soussain C, Michel-Langlet P, Mahjoubi M, et al. Metastases cérébrales du choriocarcinome chez l'homme. A propos d'un cas. Revue de la literature. Bull Cancer 1992; 79:751-7. 